\title{
Congenital limb defects in a married female population of the Rahim Yar Khan District in Pakistan
}

\author{
Hafiza Fizzah Riaz ${ }^{\oplus}$, Sajid Malik*๑
}

\section{Abstract}

Background: Congenital limb defects (CLD) have a range of phenotypes and can be a substantial cause of disability. The prevalence of CLD in the adult population of Pakistan is not well described.

Objectives: To investigate the prevalence of CLD and their associated factors in a married female population of the Rahim Yar Khan (RYK) District in Pakistan.

Methods: A cross-sectional population-based study was conducted in 4 tehsils of RYK District, and married women and girls from 22 different localities were enrolled by convenience sampling in public places and through door-to-door visits. Data regarding limb phenotype and demographic variables were obtained from participants.

Results: We enrolled 2,204 married women and girls. We found 11 participants with CLD suggesting a prevalence of $4.99 / 1,000$ (proportion: 0.005 ; $95 \%$ confidence interval $[\mathrm{CI}]<0.001-0.01$ ). Polydactyly was the most frequent $(\mathrm{n}=5$; prevalence: $2.27 / 1,000)$, followed by others in the following sequence: brachydactyly $(\mathrm{n}=4$; prevalence: $1.81 / 1,000)$, camptodactyly ( $n=1$; prevalence: $0.45 / 1,000)$, and oligodactyly $(n=1$; prevalence: $0.45 / 1,000)$. The odds of occurrence of CLD were higher in individuals originating from Khanpur tehsil (odds ratio [OR] 2.05; 95\% CI 0.3711.27), speaking languages other than Punjabi and Saraiki (OR 2.35; 95\% CI 0.24-22.80), belonging to Araien caste (OR 2.35; 95\% CI: 0.24-22.80), of a nuclear family (OR 3.35; 95\% CI 0.79-16.97), or having parental consanguinity (OR 1.87; 95\% CI 0.49-7.06).

Conclusion: Preliminary estimate of CLD prevalence in the married female sample population in RYK appears high compared with estimates from birth defects registries in other countries.

Keywords: descriptive epidemiology; limb defects; polydactyly; Southern Punjab; women's health

Congenital limb defects (CLD) are easily recognized and have long-lasting physical, psychological, and social impacts on the life of the individual. CLD occur due to failure of proper limb development during embryonic stages. Depending upon their involvement and nature, they may be classified as upper or lower limb defects, preaxial or postaxial, isolated or syndromic, and the phenotypes range from mild to severe $[1,2]$. They are grossly categorized as failure of formation or differentiation, duplications, overgrowth or undergrowth, constriction bands, or generalized skeletal abnormalities [1]. The pattern, incidence, and etiology of CLD vary greatly from population to population. In many populations, CLD are the most prevalent type of congenital anomaly after congenital heart defects. Their prevalence may range from $5-21 / 10,000$ births $[3,4]$.

*Correspondence to: Sajid Malik, Human Genetics Program, Department of Zoology, Faculty of Biological Sciences, Quaid-i-Azam University, Islamabad 45320, Pakistan, email: malik@qau.edu.pk Human Genetics Program, Department of Zoology, Faculty of Biological Sciences, Quaid-i-Azam University, Islamabad 45320, Pakistan

¿ Open Access. ๑ 2021 Riaz and Malik, published by Sciendo. (cc)BY-NC-ND This work is licensed under the Creative Commons Attribution NonCommercial-NoDerivatives 4.0 License. 
CLD generally appear as isolated entities, but may also occur in clinically recognizable associations that suggest a common developmental etiology. In a multicenter study, Rosano et al. assembled data from 11 birth defect registries and showed that the prevalence of nonsyndromic limb defects appearing with at least one major malformation was 1.3/10,000 [5]. They also reported that preaxial limb defects occurred more often with developmental disorders including microtia, esophageal atresia, anorectal atresia, and heart defects; postaxial limb defects occurred frequently with hypospadias; transverse defects appeared with craniofacial defects, ring constrictions, and muscular defects. Hence, certain CLD types may be the indicators of distinct sets of malformations $[5,6]$.

The prevalence and pattern of CLD in Pakistan is not well described $[2,7,8]$. Few published studies report neonatal dysmorphology, while CLD in the adult population remains largely unexplored. We conducted a descriptive epidemiological study to elucidate the prevalence and pattern of CLD and their associated factors in the married female population of the Rahim Yar Khan (RYK) District in Southern Punjab, Pakistan. Women and girls represent $48 \%$ of the RYK population [9] and in 1998 there were about 1 million ever-married women and girls aged 15-49 years (Population Census Organization. 1998 District Census Report of Rahim Yar Khan. Table 29. Ever Married Women (15-49 years) by age group. Statistics Division. Government of Pakistan).
RYK District is located in the southern extreme of Punjab province of Pakistan $\left(28.42^{\circ} \mathrm{N} ; 70.30^{\circ} \mathrm{E}\right)$. The District is divided into 4 administrative regions called tehsils, namely Sadiqabad, RYK, Khanpur, and Liaquatpur (Figure 1) [9]. About $80 \%$ of the district's 4.7 million inhabitants reside in rural areas. Saraiki is the predominant language followed by Punjabi and Urdu. The major caste systems are Araien, Jutt, Rajpoot, Gujjars, Malik, and Baloch. The district has an agriculture-based economy. The overall literacy rate is $33 \%$ and female literacy is even less at about 22\%. Marriages are traditionally arranged and consanguinity is $58 \%[9,10]$.

\section{Methods}

\section{Sample determination}

This study was approved by the Ethical Review Committee of Quaid-i-Azam University (No. DAS/10-854). A crosssectional study focusing on the ever-married women and girls was conducted from September 2010 to March 2011. The study was conducted in compliance with international guidelines for human research protection, including the principles outlined in the contemporary revision of the Declaration of Helsinki of 1964 incorporating the most recent (2008) and

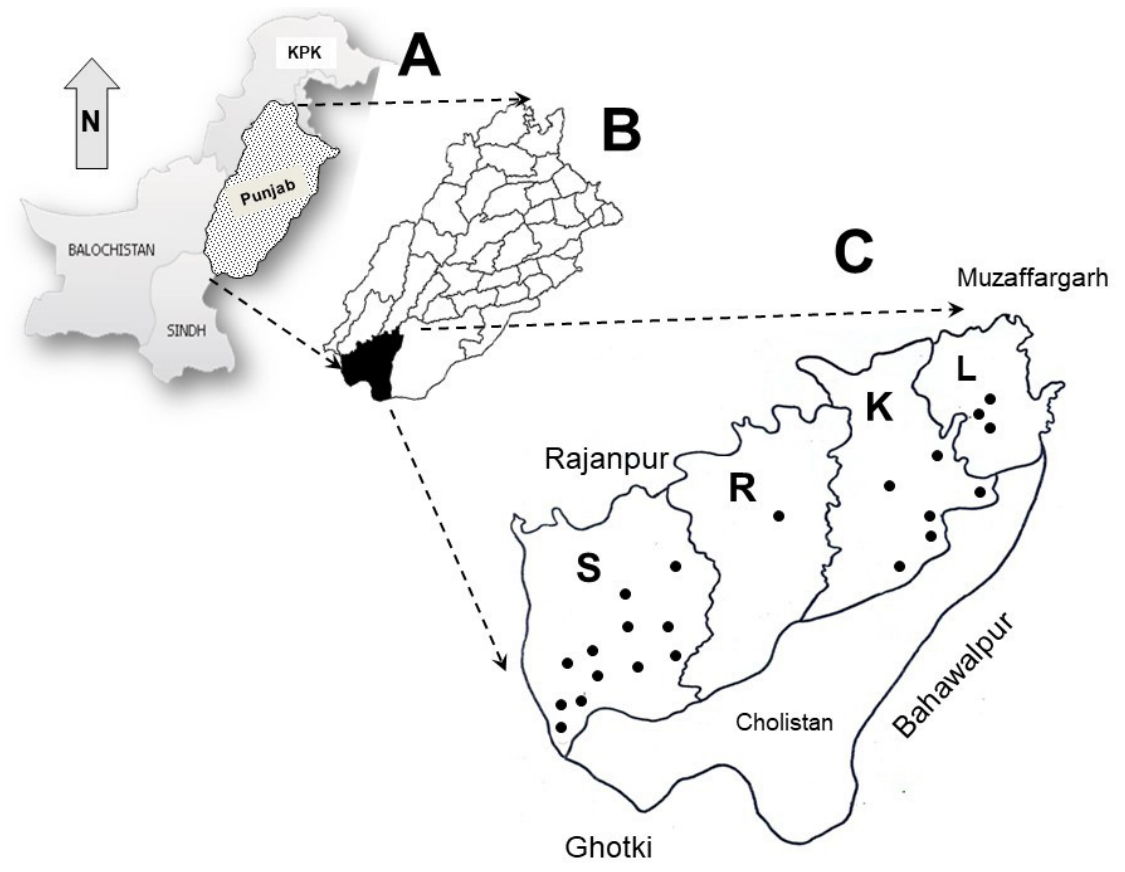

Figure 1. Map of Pakistan (A) with zoom-in view of Punjab province (B) highlighting RYK District in the far South (C) with 4 tehsils (S: Sadiq Abad; R: Rahim Yar Khan; K: Khanpur; L: Liaquatpur). Sampling sites $(n=22)$ are shown as black dots. Adapted from public domain source found at: http://www.freeworldmaps.net/asia/pakistan. RYK, Rahim Yar Khan; KPK, Khyber Pakhtunkhwa. 
earlier amendments, and Council for International Organizations of Medical Sciences (CIOMS) International Ethical Guidelines for Biomedical Research Involving Human Subjects (2002). The participants were recruited by convenience sampling from 22 different localities covering major townships and villages of the 4 tehsils of RYK District (Figure 1). The sample size was calculated from the estimated married female population using the tool available at http://sampsize.sourceforge.net/iface/with expected prevalence of $0.5 \%$ and $95 \% \mathrm{CI}$.

Field visits were conducted with the assistance of a local female health visitor who helped in obtaining documented consent and getting clinical data. The married women and girls were approached through door-to-door visits and in public places like educational institutes, hospitals, and community centers. Documented consent was obtained from each participant or her husband or husband's relatives before collecting data; assent was always obtained from the participant in any case. Only one individual from a particular household was sampled. In most households, there was just one married couple (i.e., a nuclear family). In extended families, the individual who first volunteered was enrolled. Data of individuals who were not permanent residents of the district or who did not provide complete information were excluded. We used the Strengthening the Reporting of Observational Studies in Epidemiology (STROBE) statement cross-sectional reporting guidelines [11]. Data for demographic variables and limb anomalies were recorded on a structured proforma. The individuals with limb anomalies were physically examined with the help of local medical practitioners. Case histories were taken and the previous medical record, when available, was obtained.

\section{Definitions and classification of anomalies}

CLD were classified according to the Online Mendelian Inheritance in Man (OMIM) database and the closest definition was sought in International Classification of Disease codes (ICD-10) [12, 13]. Further, detailed pedigrees were constructed for all the participants with limb anomalies.

\section{Statistical methods}

The distribution of anomalies was assessed across the biodemographic variables and $P<0.05$ was used as the cutoff for significance. Prevalence of CLD was estimated in the total sample and is represented as per 1,000 individuals. To calculate the odds ratio (OR), the minimum ratio in the respective category was taken as a reference [14]. Discrete variables were evaluated for independence by employing $\chi^{2}$ and Fisher exact tests, executed through GraphPad Prism 5 (GraphPad Software).

\section{Results}

We approached 2,662 individuals during the fieldwork, of which 458 refused to participate or provided incomplete data (i.e., 17.2\%) and 2,204 consented to participate in the study, fulfilled the inclusion criteria, and provided complete information. The response/inclusion rate was $82.8 \%$. The participants ranged 15-80 years of age (average \pm standard deviation (SD) $35.2 \pm 13.4$ years). We recruited 964 participants from Sadiqabad tehsil, 601 from RYK, 587 from Khanpur, and 52 from Liaquatpur.

We found 11 participants with limb defects, resulting in a prevalence of 4.99/1,000 married women and girls (proportion 0.005; 95\% CI <0.001-0.01). Limb anomalies were subcategorized into 4 subtypes (Table 1; Figure 2). Polydactyly was the most frequent $(\mathrm{n}=5$; prevalence: $2.27 / 1,000)$, followed by brachydactyly $(\mathrm{n}=4$; prevalence: $1.81 / 1,000)$, camptodactyly $(\mathrm{n}=1$; prevalence: $0.45 / 1,000)$, and oligodactyly $(\mathrm{n}=1$;

Table 1. Prevalence of limb anomalies in a married female population sample from Rahim Yar Khan District

\begin{tabular}{lccccc}
\hline Limb anomaly & No. & Prevalence/1,000 & 95\% Cl & OMIM & ICD-10 \\
\hline Polydactyly (all) & 5 & 2.27 & $0.72-5.31$ & 603,596 & Q69 \\
Polydactyly (postaxial A) & 4 & 1.81 & $0.48-4.67$ & 174,200 & Q69.0 \\
Polydactyly (preaxial) & 1 & 0.45 & $<0.001-2.57$ & 174,400 & Q69.1 \\
\hline Brachydactyly (all) & 4 & 1.81 & $0.48-4.67$ & 113,475 \\
Brachydactyly (brachymetatarsia IV) & 3 & 1.36 & $0.26-4.00$ & 112,800 \\
Brachydactyly type A4 & 1 & 0.45 & $<0.001-2.57$ & 114,200 \\
\hline Camptodactyly & 1 & 0.45 & $<0.001-2.57$ & M72.0 \\
Oligodactyly (thumb aplasia) & 1 & 0.45 & $<0.001-2.57$ & $\mathbf{2 . 4 8 - 8 . 9 4}$ & \\
\hline Total & $\mathbf{1 1}$ & $\mathbf{4 . 9 9}$ & & \\
\hline
\end{tabular}

$\mathrm{Cl}$, confidence interval; ICD-10, International Classification of Disease codes; OMIM, Online Mendelian Inheritance in Man. 


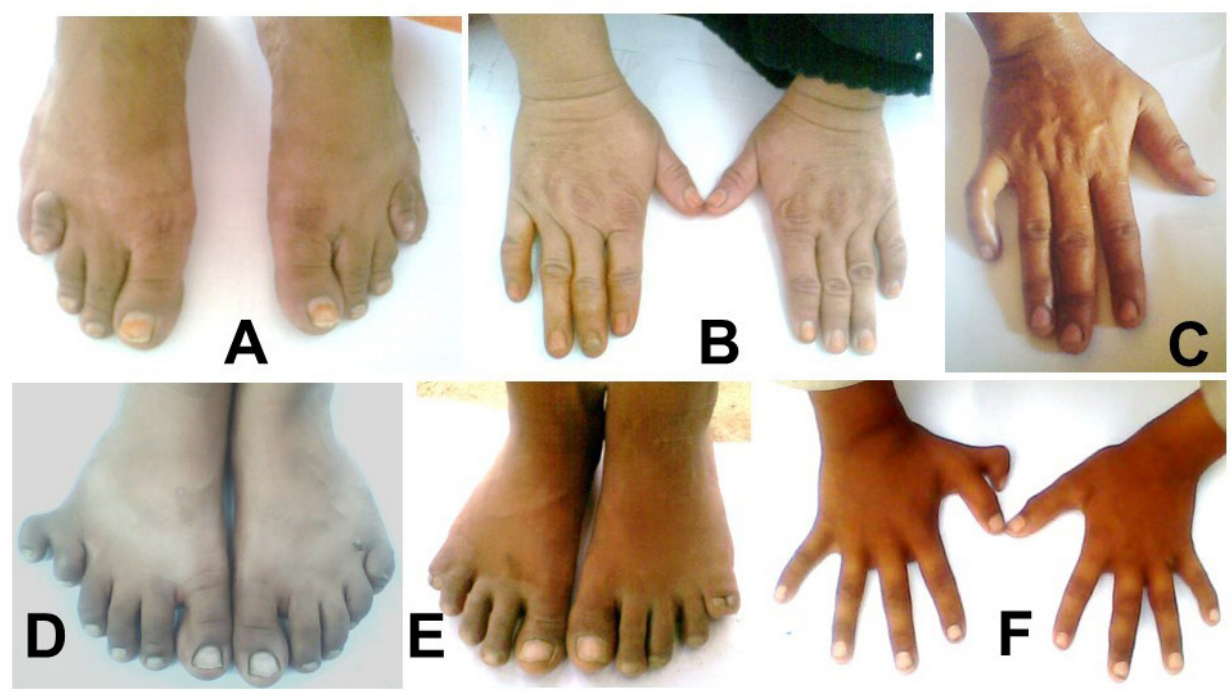

Figure 2. Phenotypic presentation of hereditary limb anomalies among the participants. (A) Bilateral brachymetatarsia of 4 th toes. (B) Brachydactyly type A4 depicting bilaterally small 2nd and 5th fingers. (C) Camptodactyly of right 5th finger. (D) Postaxial polydactyly type A of right foot. (E) Bilateral postaxial polydactyly of 5th toes (polysyndactyly of 5th to 6th toes). (F) Preaxial polydactyly (hyperdactyly) of right hand marked by the presence of a supernumerary digit. With documented informed consent for publication from each participant or with their assent and consent from their husband or husband's first degree relative.

Table 2. Characteristics of limb anomalies in a married female population sample from Rahim Yar Khan District

\begin{tabular}{|c|c|c|c|c|c|}
\hline Features & Polydactyly (all) & Brachydactyly (all) & Camptodactyly & Oligodactyly & Total \\
\hline No. of cases & 5 & 4 & 1 & 1 & 11 \\
\hline Total affected in all families & 12 & 5 & 1 & 1 & 19 \\
\hline \multicolumn{6}{|l|}{ Familial/sporadic nature } \\
\hline Familial & 4 & 1 & 0 & 0 & 5 \\
\hline Sporadic & 1 & 3 & 1 & 1 & 6 \\
\hline \multicolumn{6}{|l|}{ Laterality } \\
\hline Unilateral & 3 & 1 & 1 & 0 & 5 \\
\hline Bilateral & 2 & 3 & 0 & 1 & 6 \\
\hline \multicolumn{6}{|l|}{ Symmetry } \\
\hline Symmetrical & 2 & 3 & 0 & 1 & 6 \\
\hline Asymmetrical & 3 & 1 & 1 & 0 & 5 \\
\hline \multicolumn{6}{|l|}{ Involvement of limb } \\
\hline Upper right & 3 & 1 & 1 & 1 & 6 \\
\hline Upper left & 1 & 1 & 0 & 1 & 3 \\
\hline Lower right & 2 & 2 & 0 & 0 & 4 \\
\hline Lower left & 1 & 3 & 0 & 0 & 4 \\
\hline Total limbs involved & 7 & 7 & 1 & 2 & 17 \\
\hline
\end{tabular}

prevalence: $0.45 / 1,000)$. There were 4 individuals with polydactyly postaxial type A (prevalence: $1.81 / 1,000)$ and 1 individual with preaxial polydactyly (prevalence: $0.45 / 1,000$ ). Of those with brachydactyly, there were 3 with brachymetatarsia IV (prevalence: $1.36 / 1,000$ ) and 1 with brachydactyly type A4 (prevalence: $0.45 / 1,000$ ).
Pedigree analyses suggested that 5 CLD had familial presentations, while 6 were sporadic (Table 2; Figure 3). Anomalies in 5 participants had unilateral limb involvement and 6 were bilateral. Among those 11 participants, there were 17 affected limbs, with nearly equal involvement of upper and lower limbs (9 vs. 8). 

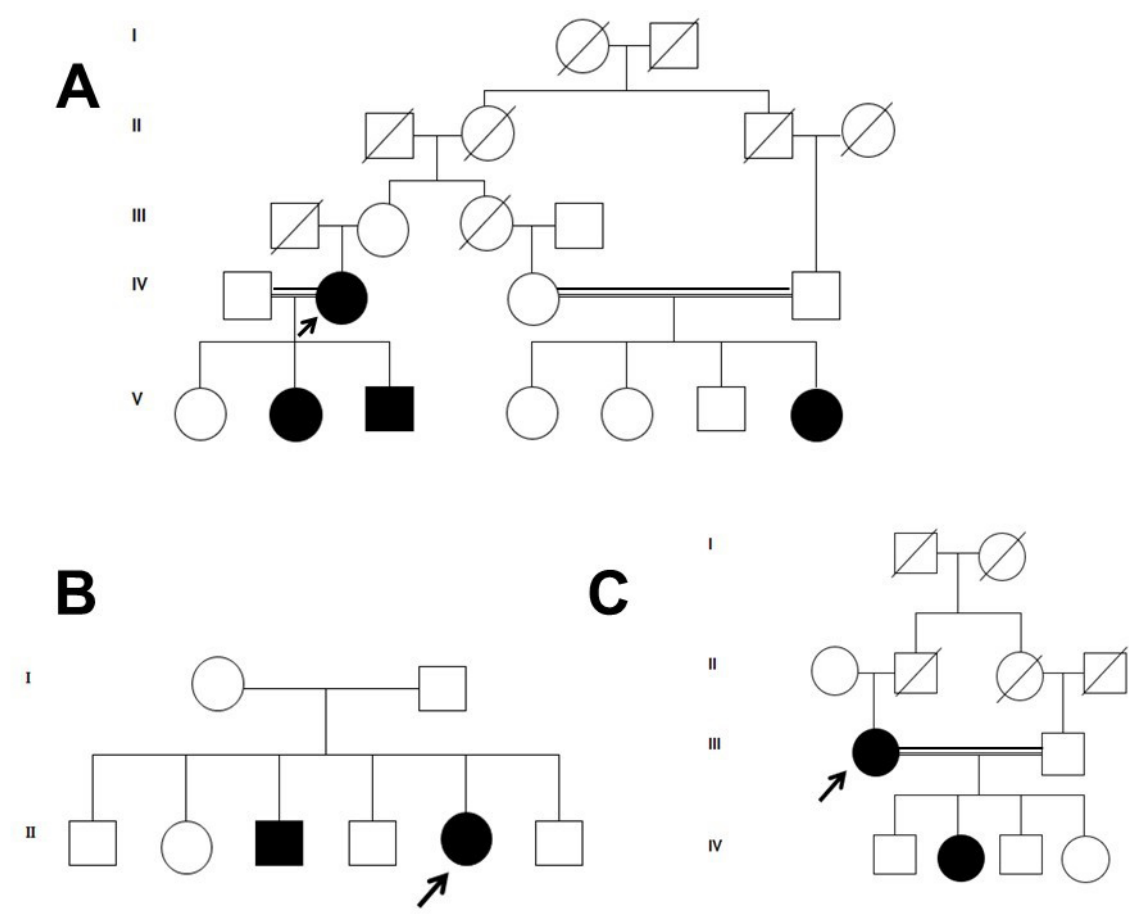

Figure 3. Representative pedigrees of familial cases. (A) Five generation pedigree showing the transmission of polydactyly in 2 independent sibships. In both sibships there were consanguineous marriages. (B) Two generation pedigree showing 2 affected sibs with brachydactyly. (C) Four generation kindred exhibiting the transmission of polydactyly in 2 generations. With informed consent for publication from each participant or with their assent and consent from their husband or a first degree relative.

The odds of the occurrence of limb anomalies were higher in individuals originating from Khanpur tehsil (OR 2.05; 95\% CI 0.37-11.27), speaking languages (which include all minor dialects $[<50]$ ) other than Punjabi and Saraiki (OR 2.35; 95\% CI $0.24-22.80$ ), belonging to Araien caste (OR 2.35; 95\% CI $0.24-22.80$ ), of a nuclear family type (OR $3.35 ; 95 \%$ CI 0.79-16.97), having parental consanguinity (OR 1.87 ; $95 \%$ CI $0.49-7.06$ ), or those who fall in the age category $>29$ years (OR 2.31; 95\% CI 0.81-6.57) (Table 3).

\section{Discussion}

The etiology of CLD is complex, ranging from purely genetic to environmental, or there may be a combination of genetic defects and environmental factors such as smoke, tobacco, chemicals, or medications [6]. CLD in Pakistan have not been widely reported in the medical literature. Minor limb defects that do not cause impairment in the limb functions are often ignored or not considered as birth defects by many pediatricians [15]. Particularly, incomplete soft-tissue syndactyly, minor polydactyly and brachydactyly, camptodactyly, and clinodactyly do not attract the attention of medical practitioners $[16,17]$.
The present study was undertaken to assess the prevalence and factors associated with limb defects in the married female population of RYK District. Based mainly on convenience sampling, this study is a pilot attempt to estimate the prevalence of limb defects in the adult population. There are no published data available that can indicate the spectrum of limb anomalies in RYK, although a few case studies of specific disease types have been published [18, 19]. Nationwide health surveys are regularly conducted in many countries to update the existing disease prevalence, assess their impact on society, and identify demographic correlates and risk factors $[4,5]$.

In the present study, there were 11 participants with 5 types of limb defects, suggesting a prevalence of 4.99/1,000 in the married female population. This estimate is high compared with other studies conducted of neonates [3, 4]. For instance, based on the data from birth defects registries in Southern Thailand, the average prevalence of CLD was 2.27 per 1,000 births (95\% CI 2.05-2.49) [20]. The most common CLD was talipes equinovarus (44.1\%), followed by polydactyly (13.9\%) and syndactyly $(9.4 \%)$. In a 30 year population-based study carried out in the Netherlands, the prevalence of polydactyly was $8.4 / 10,000$, followed by reduction defects $6.9 / 10,000$, and then by syndactyly 4.7/10,000 [4]. 
Table 3. Association of limb anomalies in sociodemographic attributes of participants

\begin{tabular}{|c|c|c|c|c|c|}
\hline Variable & Affected & Unaffected & Prevalence/1,000 & OR & $95 \% \mathrm{Cl}$ \\
\hline \multicolumn{6}{|l|}{ Tehsil } \\
\hline Rahim Yar Khan & 2 & 599 & 3.32 & Reference & \\
\hline Khanpur & 4 & 583 & 6.81 & 2.05 & $0.37-11.27$ \\
\hline Sadiqabad & 5 & 959 & 5.20 & 1.56 & $0.30-8.08$ \\
\hline Liaquatpur & 0 & 52 & 0.0 & & \\
\hline Total & 11 & 2193 & 4.99 & & \\
\hline \multicolumn{6}{|l|}{ Native dialect } \\
\hline Punjabi & 7 & 1105 & 6.29 & 2.01 & $0.52-7.81$ \\
\hline Saraiki & 3 & 953 & 3.14 & Reference & \\
\hline Other & 1 & 135 & 7.35 & 2.35 & $0.24-22.80$ \\
\hline \multicolumn{6}{|l|}{ Place of residence } \\
\hline Rural & 7 & 1409 & 4.94 & Reference & \\
\hline Urban & 4 & 784 & 5.08 & 1.03 & $0.30-3.52$ \\
\hline \multicolumn{6}{|l|}{ Family structure } \\
\hline Nuclear family & 9 & 1210 & 7.38 & 3.35 & $0.79-16.97$ \\
\hline Extended Family & 2 & 983 & 2.03 & Reference & \\
\hline \multicolumn{6}{|l|}{ Parental marriage } \\
\hline Consanguineous & 8 & 1290 & 6.16 & 1.87 & $0.49-7.06$ \\
\hline Nonconsanguineous & 3 & 903 & 3.31 & Reference & \\
\hline \multicolumn{6}{|l|}{ Education } \\
\hline Illiterate & 7 & 1464 & 4.76 & Reference & \\
\hline Literate & 4 & 729 & 5.46 & 1.15 & $0.33-3.93$ \\
\hline \multicolumn{6}{|l|}{ Age group (years) } \\
\hline$\leq 29$ & 5 & 1075 & 4.63 & Reference & \\
\hline$>29$ & 12 & 1118 & 10.62 & 2.31 & $0.81-6.57$ \\
\hline
\end{tabular}

Contingency test-statistics were nonsignificant for all variables.

$\mathrm{Cl}$, confidence interval; $\mathrm{OR}$, odds ratio

In the present study, polydactyly was the most common limb defect $(n=5)$, followed by brachydactyly $(n=4)$. A multicenter study carried out in Jefferson County, Alabama, United States, and Uppsala County, Sweden, showed that the incidence of all types of polydactyly was $0.6 / 1,000$ in female individuals with western European and Nordic ancestry [21]. More contemporary data from European birth registries (both sexes) indicates the prevalence of polydactyly ranges from 9.91 (2013) to 14.32 (2019)/10,000 live births and the average in this whole duration is $9.59(9.31-9.88[95 \% \mathrm{CI}]) / 10,000$ [22]. In an epidemiological study conducted in Sialkot District of Pakistan, limb defects were observed to have the highest representation ( $\mathrm{n}=113$; proportion: 0.47 ; $95 \%$ CI $0.41-0.53$ ) among 241 cases of congenital anomalies [2]. Postaxial polydactyly had the highest representation among limb defects $(\mathrm{n}=29)$. In another cohort of 246 cases in which congenital anomalies were ascertained with Kurram Tribal Agency of northwest Pakistan, Zahra et al. [8] found that after neurological disorders and musculoskeletal defects, limb defects were the third most common anomaly $(\mathrm{n}=52$; proportion: 0.21 ; 95\% CI $0.16-0.26)$ and among those, polydactyly was the most prevalent $(n=12)$.

In our cohort, CLD in 10 participants had isolated presentations, while in 1 participant polydactyly occurred with cleft lip (grade 2). A review of literature revealed that polydactyly appears in combination with cleft lip in various well-established syndromes like orofaciodigital syndrome V (OFD5; OMIM 174300) and orofaciodigital syndrome VI (OFD6; OMIM 277170) [12]. However, both of these syndromes also show involvement of other organ-systems. For instance, OFD5 has additional neurological features and orofacial defects including frontal bossing, hypertelorism, and lobulated tongue. By contrast, OFD6 shows additional features of short stature, failure to thrive, and various orofacial 
defects such as micrognathia, posteriorly rotated ears, and hearing loss. Included in the present data is an individual with right hand postaxial polydactyly who had undergone surgical removal of the superfluous digit. In terms of severity, only the participant with oligodactyly (thumb aplasia) can be considered as having severe CLD.

\section{Problems and prospects in conducting fieldwork}

Fieldwork and data collection in a remote and less developed areas, mostly in rural areas such as RYK District, poses several challenges for a female investigator. Apart from the logistics and travel issues, there were sociocultural concerns in the enrollment of participants and collection of data. It was difficult to get consent from married women and girls in the absence of their husbands or husbands' relatives. Due to various factors, such as illiteracy and poor orientation regarding the health/epidemiology surveys, some individuals hesitated to participate in the study. Furthermore, owing to the security issues and previous bad experience with field workers, some individuals were reluctant to give their consent. In certain instances, the subjects did not provide complete information because of internal family conflicts, particularly in the extended families. Consent approval was enhanced by involving a local resource person (i.e., female health visitor) in the field work and by describing to them the purpose of the study and the likely outcomes.

\section{Study limitations}

The present study has several limitations. First, it focuses only on the married female population, and participants were recruited based upon the availability of resource persons/female health visitors. Due to the specific sociocultural and religious norms, we were not able to approach unmarried and younger individuals. So, the results of the present convenience sampling cannot be generalized to the whole population. Studies have shown that male individuals not only have a different pattern of disorders, but also exhibit a higher morbidity than female individuals [4]. To estimate the true prevalence and incidence of morbidities, it is important to include all the population strata, which could be a point of focus of another study. Common epidemiological study designs are birth surveillance programs and total population screening, which demand ample human and logistic resources. The present work was conducted in 2010-2011, but the findings are still relevant because of the scarcity of data pertaining to this remote population. This study presents only the limb anomalies that could be easily recognized by physical examination. However, other anomalies like neurological, musculoskeletal, and metabolic disorders were not determined. Most of the participants originate from an impoverished area, where the women do not have access to antenatal ultrasound screening. Due to the limited number of affected participants, it was not possible to determine the mode of inheritance of all pedigrees; further, we cannot exclude the possibility that the few sporadic cases may have a familial nature or no genetic basis, i.e., phenocopy [22]. Limb defects of a familial nature could be the focus of future molecular genetic studies involving SNP-based genotyping, or whole genome or exome sequencing.

\section{Conclusion}

Despite several limitations, this study provides a preliminary snapshot of the prevalence of CLD in a married female sample population from RYK District. The prevalence of 4.99/1,000 appears high when compared with estimates from birth defects registries in other countries.

Author contributions. SM contributed substantially to the conception and design of the study. HFR acquired the data. SM contributed substantially to its analysis and interpretation. SM and HFR drafted the manuscript and revised it critically for important intellectual content. Both authors approved the final version submitted for publication and take responsibility for statements made in the published article.

Acknowledgments. We thank the physicians and female health visitors from the Sheikh Zayed Hospital for their contribution toward curating the data. We are grateful for the helpful comments of Professor Dr. Afsar Mian and Professor Dr. Jahangir Arshad that assisted in preparing the manuscript. This study was supported by the Higher Education Commission, Pakistan (No. NRPU/HEC/20-1607 grant to Sajid Malik) and Pakistan Science Foundation (PSF/IS/84 to recipient Sajid Malik).

Conflicts of interest statement. The authors have each completed and submitted an International Committee of Medical Journal Editors Disclosure Form for Potential Conflicts of Interest. None of the authors has any potential or actual conflict of interest to disclose in relation to the present work.

Data sharing statement. Statistical summaries of data generated for the present article are included in the published article. Further details are available from the corresponding author on 
reasonable request after deidentification from any participant whose data are included in the present report.

\section{References}

[1] Swanson AB. A classification for congenital limb malformations. J Hand Surg Am. 1976; 1:8-22.

[2] Bhatti NA, Mumtaz S, Malik S. Epidemiological study of congenital and hereditary anomalies in Sialkot District of Pakistan revealed a high incidence of limb and neurological disorders. Asian Biomed (Res Rev News). 2019; 13:49-60.

[3] Evans JA, Vitez M, Czeizel A. Congenital abnormalities associated with limb defects: a population study based on cases from the Hungarian Congenital Malformation Registry (1975-1984). Am J Med Genet. 1994; 49:52-66.

[4] Vasluian E, van der Sluis CK, van Essen AJ, Bergman JE, Dijkstra PU, Reinders-Messelink HA, de Walle HE. Birth prevalence for congenital limb defects in the northern Netherlands: a 30-year population-based study. BMC Musculoskelet Disord. 2013; 14:323. doi: 10.1186/1471-2474-14-323

[5] Rosano A, Botto LD, Olney RS, Khoury MJ, Ritvanen A, Goujard J, et al. Limb defects associated with major congenital anomalies: clinical and epidemiological study from the International Clearinghouse for Birth Defects Monitoring Systems. Am J Med Genet. 2000; 93:110-6.

[6] Gold NB, Westgate M-N, Holmes LB. Anatomic and etiological classification of congenital limb deficiencies. Am J Med Genet Part A. 2011; 155A:1225-35.

[7] Masood SN, Jamil N, Mumtaz SN, Masood MF, Muneer S. Congenital malformations in newborns of consanguineous and nonconsanguineous parents. Pak J Med Sci. 2011; 27:1-5.

[8] Zahra Q, Shuaib M, Malik S. Epidemiology of congenital anomalies in the Kurram Tribal Agency, northwest Pakistan. Asian Biomed (Res Rev News). 2016; 10:591-601.

[9] Pakistan Bureau of Statistics. District Census Report of Rahim Yar Khan [internet]. Islamabad: Government of Pakistan; 1998 [cited 2021 May 12]. Available from: https://www.pbs.gov.pk/content/ district-glance-raim-yar-khan

[10] Riaz HF, Mannan S, Malik S. Consanguinity and its sociobiological parameters in Rahim Yar Khan District, Southern Punjab,
Pakistan. J Health Popul Nutr. 2016; 35:14. doi: 10.1186/s41043016-0049-x

[11] von Elm E, Altman DG, Egger M, Pocock SJ, Gøtzsche PC, Vandenbroucke JP; STROBE Initiative. The Strengthening the Reporting of Observational Studies in Epidemiology (STROBE) statement: guidelines for reporting observational studies. J Clin Epidemiol. 2008; 61:344-9.

[12] Online Mendelian Inheritance in Man. OMIM [internet]. Baltimore (MD): Johns Hopkins University, ๑1966-2021 [cited 2021 Jan 16] Available from: https://www.omim.org

[13] World Health Organization. International Classification of Disease. ICD-10 [internet]. Geneva: WHO; 2010 [cited 2021 Jan 16] Available from: http://apps.who.int/classifications/icd10/browse/2010/en

[14] Pagano M, Gauvreau K. Principles of biostatistics. 2nd ed. Pacific Grove (CA): Duxbury Press; 2000.

[15] Malik S, Schott J, Ali SW, Oeffner F, Amin-ud-Din M, Ahmad W, et al. Evidence for clinical and genetic heterogeneity of syndactyly type I: the phenotype of second and third toe syndactyly maps to chromosome 3p21.31. Eur J Hum Genet. 2005; 13:1268-74.

[16] Malik S. Syndactyly: phenotypes, genetics and current classification. Eur J Hum Genet. 2012; 20:817-24.

[17] Malik S. Polydactyly: phenotypes, genetics and classification. Clin Genet. 2014; 85:203-12.

[18] Malik S, Riaz HF. Terminal transverse deficiency of fingers, symbrachydactyly with anonychia of toes, and congenital scalp defect: case report of a subject with Adams-Oliver syndrome. Pak J Med Sci. 2012; 28:231-4.

[19] Riaz HF, Malik S. Case report of a neonate with congenital transverse deficiency of hand. Pak J Med Sci. 2011; 27:1177-80.

[20] Jaruratanasirikul S, Tangtrakulwanich B, Rachatawiriyakul P, Sriplung H, Limpitikul W, Dissaneevate P, et al. Prevalence of congenital limb defects: data from birth defects registries in three provinces in Southern Thailand. Congenit Anom (Kyoto). 2016; 56:203-8.

[21] Finley WH, Gustavson KH, Hall TM, Hurst DC, Barganier CM, Wiedmeyer JA. Birth defects surveillance: Jefferson County, Alabama, and Uppsala County, Sweden. South Med J. 1994; 87:440-5.

[22] EUROCAT Network. Prevalence charts and tables. [internet]. Brussels: European Commission Joint Research Centre (JRC); 2021 [cited 2021 May 25]. Available from: https://eu-rd-platform.jrc. ec.europa.eu/eurocat/eurocat-data/prevalence_en

[23] Lenz W. Phenocopies. J Med Genet. 1973; 10:34-49. 